Pain Physician. 2003;6:373-375, ISSN 1533-3159

A CASE REPORT

\title{
Thoracolumbar Syndrome in Athletes
}

\author{
Joseph D. Fortin, DO
}

The thoracolumbar syndrome is a potential cause of back pain in athletes whose sport involves prolonged posturing with the hips flexed and the head extended upward. This syndrome may be overlooked and athletes are sometimes inappropriately treated for an incidental finding in the lower lumbar spine disclosed on con-

Recent studies have emphasized the importance of an accurate functional and anatomical diagnosis for the successful management of low back pain. Diagnostic attention and treatment measures are usually directed toward the lower lumbar segments where most lumbar spine pathology occurs. Consequently, thoracolumbar junction injuries are commonly overlooked in the differential diagnosis of low back pain.

The thoracolumbar junction is susceptible to extension overloading when the dorsal spine is extended while the hips are flexed and the head is extended upward. This biomechanical construct represents a potential mechanism of low back pain in athletes whose sport necessitates performing in this position.

\section{CAse Reports}

\section{Case 1}

A 35-year-old male equestrian rider presented with recurrent pain in the thoracolumbar region radiating to the right groin and right testicular area (ilioinguinal nerve territory) and right posterior iliac crest (cluneal nerve distribution). These symptoms occurred during

From Spine Technology and Rehabilitation, Fort Wayne, IN, and Indiana University School of Medicine, Fort Wayne, IN. Address Correspondence: Joseph D. Fortin, DO, Spine Technology and Rehabilitation, 7230 Engle Road, Suite 210, Fort Wayne, IN 46804. Email: fortin@pol.net

Funding: There was no external funding in preparation of this manuscript. ventional imaging studies. The clinical presentation involves point tenderness over the affected motion segment ( $T_{12}$ through L3) with pain radiating along a segmental nerve distribution (anterior or posterior rami divisions) of thoracolumbar origin. Provocative injection of the posterior joints and/or discs under image-intensifier-con- trol can provide a definitive diagnosis. Muscle balancing and stabilization exercises to counteract the postural inequities inherent to the sport usually allow for successful return to play.

Keywords: Thoracolumbar Syndrome, Biomechanics, Provocation Injection, Muscle Balance a jump take-off when the rider was "left behind" (i.e. the horse's center of gravity was abruptly ahead of the rider's until their respective hind quarters collided). The premorbid history disclosed occasional vague, fleeting and focal thoracolumbar pain, associated with training sessions. On physical examination, the symptoms were reproducible upon spinal extension, especially when combined with right side bending.

Plain films revealed some canting of the discovertebral (anterior intervertebral) joint upon flexion(1) and a Type II biomechanical dysfunction(2) upon coronal bending. A MRI demonstrated mild decrease in nuclear signal intensity of the L2-3 disc (on sagittal T2 weighted images) and a slight, global prominence of the annulus fibrosis but was otherwise unremarkable. There was no indication of focal disc prolapse or neurocompression.

Discography was performed on the intervertebral discs from L1-2 through L3-4. Facet joint arthrography was performed on the right L1-2 through L3-4 joints.

There was no pain provoked upon distending the L1-2 and L3-4 discs. The nucleograms showed no annular fissures or other annular pathology such as disc prolapse. In contrast, distention of the L2-3 discs provoked intense thoracolumbar, groin and testicular pain (concordant with the symptom complex). Subsequent instillation of $1.0 \mathrm{cc}$ of $0.75 \%$ Bupivacaine into the L2-3 disc provided relief of this pain (VAS 0-10 pre-procedure 7 to post- procedure 2) consistent with the duration of the anesthetic. The L2-3 nucleogram revealed multiple mid to outer anterior circumferential fissures which extended through the posterolateral corner of the disc into the epidural space and right L2 epiradicular sheath, suggesting a torsion injury(3). Parenthetically, the L2 nerve roots contribute to the ilioinguinal nerve.

Similarly, the right L1-2 and L34 posterior joint injections provoked no pain upon distention. Arthrography of these joints revealed normal appearing capsules. Distention of the right L2-3 posterior joint provoked thoracolumbar pain, which radiated in a familiar distribution over the posterior iliac crest, commensurate with the cluneal nerve pathway. Significant pain relief (VAS 0-10 pre-procedural 5 to post-procedure 1) was reported with the instillation of $0.5 \mathrm{cc}$ of $0.75 \% \mathrm{Bu}-$ pivacaine. The right L2-3 arthrogram revealed a tortuous and redundant capsule with a large, boggy superior recess.

This patient was treated with therapeutic exercise to address the altered thoracolumbar mechanics.

\section{Case 2}

A 29-year-old professional hockey player presented with refractory aching discomfort in the thoracolumbar region. The onset was insidious and his symptoms were exacerbated upon assuming his characteristic playing stance. Upon physical examination, there was exquisite tenderness upon anterior translation of the L1 vertebral segment and over the left L1- 
2 facet joint. Range of motion and neuromuscular examinations were intact.

A Single Photon Emission Computed Tomography (SPECT) scan of the thoracolumbar spine disclosed increased uptake over the left L1-2 posterior joint. A left L1-2 facet injection reproduced the patient's usual symptoms and anesthetic blockade allowed complete mitigation of the pain response.

The patient ultimately underwent successful radiofrequency denervation of the medial branches of the dorsal rami which innervate the aforementioned joint.

\section{Discussion}

Sports which necessitate repetitive spinal loading with simultaneous hip flexion and neck extension concentrate forces across the transitional thoracolumbar junction. Athletes in sports such as weight lifting, equestrian competition, hockey and football (especially linemen and linebackers) may most susceptible to thoracolumbar injury.

The thoracolumbar spine is vulnerable to extension overload injury when hip flexion is combined with the head tilted backward and upward (viewing the playing field or assuming an erect posture from a crouched one). There is relatively little extension that can occur in the thoracic spine in any position due to innate biomechanical limitations. No extension is available in the lumbosacral spine when the hips are flexed and little in the cervical spine when the head is already extended backward(4). Thus, the thoracolumbar junction becomes a pivotal region for further extension loading. This disparity in strain mechanics between the thoracic and lumbar spine can set the stage for dysfunction $(4,5)$.

When the posterior elements of this area are overloaded, the inferior articular process may impact upon the subadjacent lamina and distract the contralateral posterior joint capsule causing pain (6). Additional extension is blocked by bone contact. Future extension can cause the spinal segment to rotate around the impacted articular process producing torque upon the disc. Excessive torque can cause circumferential tears in the annulus, especially in the posterolateral corner where the disc is most vulnerable to such force (3).

The differential diagnosis of nonspecific thoracolumbar pain is extensive (7). The non-musculoskeletal causes are beyond the scope of this discussion. They include: spinal tumors, retroperitoneal processes such as renal disease, primary or secondary neurogenic causes such as neurofibroma, vascular abnormalities such as abdominal aortic aneurysm, and any infiltrative or inflammatory process.

The musculoskeletal entities to consider for the cause of back pain include: compromise of the anterior spinal nerves or posterior primary rami of thoracolumbar origin, pathology of the thoracolumbar zygapophyseal joints and/or their joint capsules, thoracolumbar disc disruption, congenital malformations, degenerative processes and fibromyalgia. It is important in patients subjected to significant trauma to rule out vertebral fracture (8) (i.e. Chance, burst or compression type) and/or spinal instability.

An athlete's presentation of thoracolumbar pain may superficially appear ambiguous. A careful history, however, often discloses an appropriate mechanism of injury as well as pain distributed in a thoracolumbar pattern.

Maigne provided a hallmark description of the physical findings for the thoracolumbar syndrome (9). The pathology often includes the thoracolumbar segments from T11 to L3. Exquisite tenderness is noted on direct palpation of one or more thoracolumbar segments (i.e. T11 to L3). Pinching and rolling the skin over the iliac crest (cluneal nerve)(10), inguinal canal (inguinal nerve) or greater trochanter (lateral perforator nerve) may elicit an immediate, local dysethetic re- sponse (11).

Imaging (plain films, MRI, CT and radionuclide scans) and selective injection studies (discography, posterior joint blocks) should be employed as indicated to corroborate clinical findings $(12,13)$ These tests may aid in establishing anterior column versus posterior element pathology or in ruling out visceral, vascular, infectious, infiltrative or neoplastic disorders.

Plain film stress views of the thoracolumbar region will document gross segmental instability (2). Conventional CT and axial sections on MRI of the lumbar spine most often focus on the L3-4 to L5/S1 motion segments (13). Consequently, it is common for the thoracolumbar syndrome to be disregarded in light of an incidental imaging finding in the lower lumbar spine (12). Lumbosacral, sacroiliac, inguinal or hip pathology should not be mistaken for the thoracolumbar syndrome.

Treatment consists of a four-phase functional restoration program as shown in Table 1. The focus is placed on restoration of the correct biomechanics of the thoracolumbar junction.

Patients are educated on the susceptibility of thoracolumbar injury from posturing with the head tilted upwards, thoracic spine extended and hips flexed. This awareness increases the patient's understanding of the mechanism of injury, enhances interest in the rehabilitative process and empowers the patient to take preventative measures to avoid re-injury.

\section{Table 1. The functional restoration program}

1. Decrease pain and inflammation

Ice and electrical stimulation

Nonsteroidal anti-inflammatory agents

Postural education

Myofascial therapy

2. Restore range of motion

Manual medicine

Flexibility and muscle balancing

Dissociative movement therapy (beginning)

Elementary stabilization

Gait mechanics

3. Improve strength and stability

Intermediate and advanced stabilization

Proprioceptive retraining

Dissociative movement therapy (intermediate and advanced)

Plyometrics

Resistive exercises/weight training

4. Return to work/return to play

Task and/or work specific activities 
Manual medicine is aimed at restoring motion in hypomobile motion segments. Manipulation would be contraindicated at the level of a hypermobile motion segment because it may cause injury (e.g. further disruption of annular tears). A detailed discussion of manual medicine is beyond the scope of this report and can be found in other sources (1).

Muscle balancing first involves identification of abnormally lengthened or shortened muscles which may have lead to the injury or are a result of muscle substitution patterns after the injury. Muscles are then trained at the proper position to provide correct functional mechanics.

Muscle stabilization involves muscle strengthening to reinforce hypermobile segments and modify the mechanical stressors of movement. Examples are eccentric strengthening of the transversus abdominis to control thoracolumbar trunk rotation and the thoracolumbar multifidus to control trunk extension.

\section{CONCLUSION}

Consideration of the cause of low back pain usually centers on the lower lumbar segments. Consequently, the thoracolumbar syndrome may be overlooked. The thoracolumbar junction is

susceptible to extension overload injuries when the spine is positioned in such a way that limited extension is available in the cervical or lumbosacral regions. Important diagnostic tools include an understanding of the mechanism of injury and diagnostic studies, including imaging modalities and pain provocation injections. Rehabilitation is directed toward restoring proper thoracolumbar mechanics.

\section{Author Affiliation: \\ Joseph D. Fortin, DO \\ Medical Director \\ Spine Technology and Rehabilitation, \\ Clinical Professor \\ Indiana University School of Medicine \\ 7230 Engle Road, Suite 210 \\ Fort Wayne, IN 46804 \\ E-mail: fortin@pol.net}

\section{References}

1. Greenman PE (Ed). Principles of Manual Medicine, Williams and Wilkins, Baltimore, 1989, pp 49-70.

2. Kirkaldy-Willis WH, Burton CV (Eds). Managing low back pain, 2nd Edition, Churchill-Livingstone, New York, pp 203223.

3. Farfan HF, Cossette JW, Robertson GH et al. The effects of torsion on the lumbar intervertebral joints: The role of torsion in the production of disc degeneration. J. Bone Joint Surg (Am) 1970; 52:468-497.

4. White AA, Panjabi MM. The basic kinematics of the human spine. A review of past and current knowledge, Spine 1978; 3:1220.

5. White AA, Panjabi MM. Clinical biomechanics of the spine. Lippincott, Philadelphia 1978, pp 257-265.

6. Yang KH, King Al. Mechanism of facet load transmission as a hypothesis for low back pain, Spine 1984; 9:557-565.

7. McNab I (Ed) Backache. Williams and Wilkins, Baltimore, 1977.

8. Yashon D. Spinal Injury. Prentice-Hall, Norwalk, CT, 1986, pp 219-220.

9. Maigne R. Low back pain of thoracolumbar origin, Arch of Phys Med and Rehabil , 1980; 61:389-395.

10. Maigne R. Trigger point of the posterior iliac crest: Painful ileolumbar ligaments insertion for cutaneous dorsal ramus pain? An anatomic study, Arch of Phys Med and Rehabil 1991; 72:734-737.

11. Kirkaldy-Willis WH, Burton CV (Eds). Managing low back pain. 3rd Edition, Churchill-Livingstone, New York, 1992, pp 225-241.

12. Fortin JD. Enigmatic Causes of Spine Pain in Athletes. In Watkins, RG (ed) The Spine in Sports. Mosby Year-Book, Chicago, 1994.

13. Herzog RJ. State-of-the-art imaging studies of spinal disorders. State-of-the-art reviews, 1990, 4:224-234. 
